\title{
Charles Bonnet Syndrome in an Elderly Patient Following Cataract Surgery
}

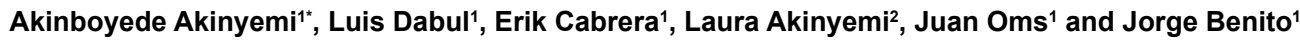 \\ ${ }^{1}$ Department of Psychiatry, Larkin Community Hospital, South Miami, FL, USA \\ ${ }^{2}$ Design Neuroscience Center, Doral, Florida, USA
}

\begin{abstract}
Introduction: The incidence and prevalence of visual impairment among the elderly population in the United States progressively shows an increase. The escalating trend among the geriatric population is relevant not only to ophthalmologists, but surprisingly relevant to Psychiatrists. Advancements in medicine have made state of the art surgical interventions available for individuals with visual impairments. However, Psychiatrists needs to be adequately informed of rare signs pointing towards complex ocular misperception-related disorders among the geriatric population which seem like psychotic episodes. One of such complex diagnoses includes Charles Bonnet syndrome.
\end{abstract}

Case presentation: This case report describes the constellation of presenting symptoms, relevance of a visual field examination and appropriate discharge disposition in an elderly female with a past medical history of cataracts status-post surgical extraction. Presenting symptoms included paranoia, agitation, visual hallucinations, and irritable mood despite treatment with multiple psychotropic medications at home. The working diagnosis of Charles Bonnet Syndrome was effectively utilized in managing this patient.

Conclusion: As the incidence of visual impairment and cataract surgeries increase consistently in the United States of America, there is an increased need for Psychiatrists to be more aware of broad neuro-ophthalmology differential diagnoses especially when assessing the behaviors of geriatric patients.

Keywords: Charles bonnet syndrome; Visual hallucinations; Cognitive decline; Visual impairment; Antipsychotic agents; Cholinesterase inhibitor

\section{Introduction}

According to the United States Census Bureau (2016), the world population is witnessing a trend of increasing longevity resulting in a corresponding growth of the geriatric population (65 years and older) [1]. The World Health Organization (WHO) projects that between 2015 and 2050, the world's geriatric population would double from $12 \%$ to $22 \%$ with an estimated increase from 900 million to 2 billion elderly people globally [2]. As a result of this trend, challenges face the medical field given that the geriatric population often undergoes age related challenges leading to decreased general functioning [2].

Mental health issues among the geriatric population mainly occur due to abnormal age-related degenerative brain changes with the most common issues being neurocognitive disorder including Alzheimer's dementia with behavioral disturbance and depressive disorder [2]. Visual impairment especially age-related macular degeneration, cataracts, diabetic retinopathy and glaucoma also commonly occur among the elderly amidst other serious medical and neurological conditions [3]. With multiple medical conditions co-occurring among the elderly population, it becomes daunting for Psychiatrists to determine and establish accurate diagnoses and prescribe the single best treatment [4]. Challenges posed to developed countries including the United States entails the management of the geriatric population as these groups of people often is on multiple medications by the time they are initially seen by a Psychiatrist [5]. This case report brings to view the thin line separating the diagnosis of an obvious psychiatric condition versus an actual ophthalmological ailment. We successfully utilized the working diagnosis of Charles Bonnet syndrome (CBS) in the management of an elderly patient with visual perceptual distortions post cataract extraction. With the growing global geriatric population, we hypothesize that similar cases as depicted here would intermittently pose challenges to Psychiatrists at various geographical locations globally.
CBS was first described in 1760 by a Swiss philosopher - Charles Bonnet, who observed and documented a constellation of symptoms of visual hallucinations and visual impairment in his own grandfather. The term CBS was later coined by another Swiss scientist - George De Morsier in the year 1967 [6]. Visual hallucinations are defined as perceptions in the visual fields without external stimuli [7]. Mocellin et al. in year 2006 described some mechanisms through which visual hallucinations occur, proposing that lesions in the brainstem may disrupt inhibitory serotonergic raphe nucleus inputs leading to excitation of the dorsal lateral geniculate nucleus (LGN) causing deregulation of retinal inputs resulting in visual hallucinations [8]. While there are variations to the presentation and progression of CBS, it should be noted that visual impairment and visual hallucinations are the key history component that must be present [9].

In the United States, the number of ophthalmological procedures is increasing among the geriatric population, especially due to cataracts and glaucoma. It is estimated that one third of patients in the geriatric population would have at least one problem affecting visual acuity by age 65 years [10]. Among these vision impairments, cataracts are the most common cause of blindness globally [10]. However, with readily available surgical cataract extraction in the United States, it is estimated by the National Eye Institute that out of 24.4 million Americans that have cataracts, 3.6 million underwent cataract procedures in 2015 alone $[11,12]$. By year 2050, the amount of Americans with cataract

*Corresponding author: Akinboyede Akinyemi, PGY-2 Psychiatry Resident, Department of Psychiatry, Larkin Community Hospital, Florida, USA, Tel: 305-2847608; Fax: 305-284-7787; E-mail: aakinyemi@larkinhospital.com

Received: July 06, 2017; Accepted: July 17, 2017; Published: July 24, 2017

Citation: Akinyemi A, Dabul L, Cabrera E, Akinyemi L, Oms J, et al. (2017) Charles Bonnet Syndrome in an Elderly Patient Following Cataract Surgery. J Psychiatry 20: 414. doi:10.4172/2378-5756.1000414

Copyright: (c) 2017 Akinyemi A, et al. This is an open-access article distributed under the terms of the Creative Commons Attribution License, which permits unrestricted use, distribution, and reproduction in any medium, provided the original author and source are credited 
is projected to increase by $50 \%$ increasing from 24.4 million to approximately 50 million, with most cases being of the white race. In relation to this case report, Hispanic-Americans are expected to have the most rapid increase in prevalence from 1.76 million cataract cases to 9.51 million [11]. With these estimates, we therefore project that there would more similar elderly patients with visual hallucinations post cataract extraction presenting for psychiatric assessments throughout the United States.

\section{Case Presentation}

76 year old Cuban-American Female presented to our emergency department (ED) involuntarily under Florida Baker Act initiated by law enforcement officials for involuntary mental health assessment. Upon initial contact with patient, she expressed her dissatisfaction over being at the ED. She mentioned that she was at her residence when the police suddenly came to arrest her. While she appeared confused regarding being in the hospital, she stated her own bio psychosocial history and denied having perceptual disturbances to auditory, visual or olfactory modalities. She denied having suicidal or homicidal ideations. Patient also denied having any past psychiatric history. Overall, her mental status assessment was unremarkable except for irritable mood, anxiety and anger over being assessed in the ED. Given the circumstances surrounding her involuntary presentation, angry mood and evidence of minimization of symptoms, collateral information was obtained from law enforcement officers and patient's son who were present at the ED.

According to law enforcement officers, a call was received to respond to the residence of an elderly female who was attempting to jump out of a moving vehicle and refusing to go for medical assessment contrary to her son's recommendation. When she was approached at her apartment, she was unable to recognize being at her own residence and she became agitated. Thereafter she tried to walk into moving traffic in her neighborhood. She was thus placed under the Baker Act and brought to the hospital for mental health assessment due to imminent self-injurious behavior to self and others.

Past medical and psychiatric history was retrieved from patient's son who stated that she was without any mental illness until age 74 (2 years preceding ED presentation) when she was found inappropriately undressing herself at a public location. At that time, she had her first psychiatric assessment and hospitalization leading to the diagnosis of unspecified psychosis. During that prior assessment, non-contrast brain Computed Tomography (CT) was performed and showed old regions of mini ischemia without any reported significance or cognitive impairment. According to her son, she was prescribed Quetiapine $12.5 \mathrm{mg}$ orally at bedtime for psychosis and insomnia. Response to medication was sufficient for 3 - 4 months after and dosage was increased effectively to $25 \mathrm{mg}$ orally at bedtime. Patient reportedly was without any further inappropriate behavior.

Patient's son mentioned that she was diagnosed with cataracts and glaucoma a few weeks following the aforementioned incident and she was scheduled for bilateral cataract extraction which did not occur until approximately 8 months later. One week after cataract extraction, client had similar presentation in which she became confused and was found talking to her and also making reference to some images that could not be appreciated by others. She reportedly had difficulty sleeping at night and would soliloquize throughout the night but sleep during the day. Her symptoms were managed at an outpatient psychiatric facility with Alprazolam $0.25 \mathrm{mg}$ at bedtime for nocturnal insomnia with improvement. A family member was designated to stay with patient at her apartment due to decline in function due to visual hallucinations, paranoia and delusions of theft of personal belongings. In the absence of the designated family member, within a period of one week, patient became more paranoid and developed visual hallucinations of images which she stayed up at night holding conversations with. In an attempt to provide safety for patient, she was advertently moved to her son's house. While at the new location, her visual hallucinations worsened to the point that she became agitated stating there were too many people around her while in reality there was no one else around. She was evaluated by a psychiatrist a few days prior to ED presentation due to frequent visual hallucinations and paranoia towards her and accusing her son of attempting to hurt her. Patient was started on Risperidone $0.25 \mathrm{mg}$ orally twice a day by another facility prior to ED presentation.

While on Quetiapine $25 \mathrm{mg}$ orally at bedtime, Alprazolam 0.25 mg orally at bedtime and Risperidone $0.25 \mathrm{mg}$ orally twice a day, the intensity of her visual hallucinations, paranoia and delusions in which patient believed that her personal items were being stolen by her son persisted. Patient requested for her son to take her back to her own residence and when she was being taken back, she became agitated and confused thus requiring law enforcement intervention which led to her ED presentation for involuntary psychiatric assessment.

Physical examination was performed at the ED with special focus towards patient's cognition and visual fields based on the above history. There was evidence of significantly diminished visual acuity bilaterally with Snellen chart as she was completely unable to read any of the alphabets. The findings were in the corresponding visual acuity consistent with legal blindness with a visual acuity of $>20 / 200$ recorded bilaterally. In confirmation, patient was unable to identify objects placed in front of her and she was found confabulating and repeating the wrong answers even when obviously incorrect. She appeared anxious throughout encounter with noted rapid speech however non -hyper verbal and easy to redirect. Thought content showed helplessness, hopelessness and catastrophizing but without suicidal or homicidal ideations. Insight was limited and judgment was grossly impaired given the fact that she continuously minimized her visual perceptual symptoms even while there was clear evidence of confabulation. She appeared her stated age, calm, cooperative, oriented to person, place and time. When patient was left alone in a room in the ED, she was noted to be soliloquizing and appeared to be moving as if she was picking some objects from the air. She did not display this behavior when there was someone with her in the room.

Urine toxicology was positive for benzodiazepine only; complete blood count and Comprehensive metabolic panel were unremarkable. Laboratory tests in the ED showed unremarkable findings with Glucose $91 \mathrm{mg} / \mathrm{dL}$, Blood Urea Nitrogen (BUN) $16 \mathrm{mg} / \mathrm{dL}$, Creatinine $0.73 \mathrm{mg} /$ $\mathrm{dL}$, Sodium $(\mathrm{Na}) 136 \mathrm{mEq} / \mathrm{L}$, Potassium $(\mathrm{K}) 4.3 \mathrm{mEq} / \mathrm{L}$, Chloride $(\mathrm{Cl})$ $103 \mathrm{mEq} / \mathrm{L}$, Blood alcohol level $<14.0 \mathrm{mg} / \mathrm{dL}$, Glomerular filtration rate (GFR) $82 \mathrm{~mL} / \mathrm{min}$ per $1.73 \mathrm{~m}^{2}$, WBC $7.9 \times 10^{9}$ per liter, RBC 5.10 $\times 10^{6} / \mu \mathrm{L}, \mathrm{HGB} 15.1 \mathrm{~g} / \mathrm{dL}, \mathrm{HCT} 45.6 \%$, \%Neutrophil 62.4. Urinalysis showed +1 leukocyte esterase with negative nitrites. Vital signs were within normal limits. CT- Brain without contrast showed diffuse brain atrophy with periventricular white matter hypodensities secondary to chronic ischemic changes, bilateral calcification of the basal ganglia, and ventricular dilatation most notably of the third ventricle. Right eye lens could not be visualized on imaging.

Given the history findings of visual impairment, agitation requiring involuntary commitment, collateral information showing evidence of paranoia, visual hallucinations, absence of auditory hallucinations, intact sensorium and physical examination findings showing visual impairment, a diagnosis of CBS was utilized as a working diagnosis. In 
order to prevent harm to self and others, optimize medications regimen and investigate psychotic disorders or other cognition related disorders, inpatient psychiatric hospitalization was initiated. Patient was started on Rivastigmine $4.6 \mathrm{mg} / 24$ hour patch, continued Alprazolam $0.5 \mathrm{mg}$ orally at bedtime to prevent benzodiazepine withdrawal, olanzapine 5 mg orally at bedtime was started for psychotic features. Risperidone $0.25 \mathrm{mg}$ and Quetiapine $12.5 \mathrm{mg}$ were discontinued to prevent multiple antipsychotic therapies. A comprehensive neuropsychological battery was ordered to delineate cognitive function due to worsening cognitive function based on obtained history and collateral information retrieved.

Neuropsychological evaluation revealed impairment in areas of attention, recent memory, executive function planning and/or problemsolving. On the Control Word Association test and Sentence Repetition test-form $\mathrm{A}$, the patient attained a percentile score of $6 \%$ on both, suggesting a moderate impairment for her ability to find words when engaged in a verbal task and process incoming verbal information with the help of both attention and concentration. Intermediate, long-term memory and orientation were preserved without deficits. Based on assessment in the inpatient behavioral health unit, focus was directed towards management of sleep pattern and reorientation of patient to her current situation. Psychotropic medications including Olanzapine 5 $\mathrm{mg}$ and Rivastigmine were discontinued. Patient continued Alprazolam $0.5 \mathrm{mg}$ orally at bedtime and was able to sleep without difficulties. She came to accept that the images she visualized were not real on day 7 of hospitalization and continued with ophthalmology follow up to improve visual function.

Upon further stabilization, she was discharged to an assisted living facility (ALF) with the aim of establishing a structured environment with assistance with medication administration and prevention of risks of self-harm due to negligence or neglect. A follow up phone call to ALF revealed further improvement in patient's symptoms without any delusions, paranoia or visual hallucinations. She continued to take Alprazolam $0.5 \mathrm{mg}$ orally at bedtime which was tapered off over a 3 month period and she followed up with Psychiatrist and Ophthalmologist. Her visual field testing was reported to be at 20/50 on the left as noted on follow up evaluation with ophthalmologist versus 20/200 at the time of presentation to the Emergency Department.

\section{Discussion}

This case report represents the typical presentation of psychotic features in an elderly patient with symptoms that could be easily misdiagnosed as dementia with behavioral disturbance or psychosis. The differentiating symptom which stands unique is the presence of visual impairment in a patient with mild cognitive decline; therefore broad differential diagnoses has to be considered when there is significant visual deficit among elderly patients presenting in this manner. CBS is a condition that occurs predominantly among the geriatric population with an average age of 76.3 years according to a 2004 study by Tan et al. [13]. Coincidentally, the geriatric population carries a large amount of individuals diagnosed with various forms of cognitive impairment [13].

In an experiment performed by Merabet et al. [14] using subjects with normal visual fields and normal cognitive function, the subjects were blindfolded for 96 hours. Surprisingly, they began reporting varying intensities of visual hallucinations within 24 hours after the onset of the experiment [14]. This experiment's findings further reinforce the relevance of visual deficits and how patients with visual impairments can be easily diagnosed as having psychosis and treated without further physical examination and radiological imaging.
CBS is a clinical diagnosis made based on a constellation of symptoms, proper monitoring of case presentation sequence and exclusion of other diseases [9]. As seen in literature review, CBS does not concurrently occur with cognitive deficits [15]. As evidence by the presence cognitive impairment and hallucinations among experimentally blindfolded individuals, it is extremely difficult to include cognitive impairment as an eliminating factor when considering CBS as a differential diagnosis in patients presenting with visual hallucinations because visual impairments can negatively impact cognition [14]. Teunisse et al. [15] came up with diagnostic criteria for CBS based on a study conducted on 505 visually handicapped individuals. The following was resulted as criteria for diagnosing CBS: one complex visual hallucination within a 4 week period, a period between the first and the last hallucinations exceeding 4 weeks, full or partial retention of insight to the unreal nature of the hallucinations, absence of delusions and absence of hallucinations in other sensory modalities $[15,16]$. Based on the aforementioned, our patient met the criteria with a clear presentation of symptoms.

As noted in this case report, there were no psychiatric or cognitive changes until age 74 . The first sign of mental status change diagnosed as psychosis was at age 74. A few months following initial psychotic episode, patient was diagnosed with cataracts which gives room to believe that visual changes contributed to her initial presentation of "psychosis" given that there were no other mental status changes. CBS is an easily missed diagnosis and the main point that can be highlighted in this case as seen always in CBS is the fact that there were visual changes causing significant impairment and mental status changes [16].

Despite the evidence of cognitive impairment as shown by neuropsychological testing, cognitive symptoms were overshadowed by visual hallucinations which did not respond to Risperidone and Quetiapine. Also, the cognitive symptoms did not respond to Rivastigmine rather resulted in an exacerbation of visual hallucinations and worsening confusion. In patients with psychotic disorders, antipsychotic medications become effective within hours to days causing noticeable relief of symptoms [17]. The absence of neuroleptic treatment response in this patient suggests a wrongful diagnosis of a psychotic disorder. Further evidence showed that the eventual discontinuation of all antipsychotic medications led to the improvement of visual hallucinations and psychosis-like symptoms which further supports a diagnosis of CBS.

Rivastigmine is a cholinesterase inhibitor which functions by slowly reversibly binding to and inactivating acetylcholinesterase (AChE) or butyrylcholinesterase (BuChE) [18]. As reported on patient's neuropsychological evaluation, there was worsening of cognition in the areas of attention, short term memory and executive functioning while on Rivastigmine. It would have been expected otherwise that initiation of Rivastigmine would improve patient's cognition but contrarily, there was worsening of hallucinations. Discontinuation of Rivastigmine contributed to improvement of cognition in this patient.

Alprazolam is a triazolobenzodiazepine which enhances gammaaminobutyric acid (GABA) effects. It reaches a peak serum level within 0.7 to 2.1 hours of administration and possesses a serum half-life of between 12 to 15 hours [19]. In this case, there was good outcome from utilization of short-acting benzodiazepine without exacerbation of confusion or hallucinations. Thus, we suggest that alprazolam may be beneficial in the treatment of insomnia in patients with CBS if used in a short term of 3 months and tapered off to prevent dependence. While this is not recommended traditionally in the elderly population, 


\begin{tabular}{|c|c|c|c|c|}
\hline & Charles Bonnet Syndrome [27] & $\begin{array}{l}\text { Neurocognitive disorder } \\
\text { (Alzheimer's) [26] }\end{array}$ & Psychotic disorders [25] & Anton Babinski syndrome [22] \\
\hline Initial symptoms & Any form of Visual impairment & Forgetfulness & $\begin{array}{l}\text { Avolition } \\
\text { Alogia }\end{array}$ & $\begin{array}{c}\text { Brain injury - traumatic, infectious or } \\
\text { vascular }\end{array}$ \\
\hline Age of onset & $\begin{array}{l}\text { Variable but more in elderly } \\
\text { population }\end{array}$ & Elderly & Variable & Variable \\
\hline Progression & Gradual & Gradual & Rapid - Gradual & Gradual \\
\hline $\begin{array}{l}\text { Radiological findings } \\
\text { (CT) }\end{array}$ & None & $\begin{array}{l}\text { May have diffuse cortical } \\
\text { atrophy }\end{array}$ & Non-specific & $\begin{array}{l}\text { Any form of damage affecting the occipital } \\
\text { lobe }\end{array}$ \\
\hline Perceptual disturbances & $\begin{array}{l}\text { Visual hallucinations } \\
\text { Delusions }\end{array}$ & $\begin{array}{c}\text { Could have confabulation/ } \\
\text { delusions }\end{array}$ & $\begin{array}{l}\text { Auditory or visual } \\
\text { Hallucinations } \\
\text { Delusions } \\
\text { Disorganized speech/ } \\
\text { behavior } \\
\text { Catatonia }\end{array}$ & $\begin{array}{l}\text { Visual Hallucinations } \\
\text { Delusions } \\
\text { Anosognosia }\end{array}$ \\
\hline Treatment & $\begin{array}{c}\text { Treat eye problem } \\
\text { CBT } \\
\text { Provide reassurance } \\
\text { Provide adequate supervision }\end{array}$ & $\begin{array}{l}\text { Acetylcholinesterase inhibitor or } \\
\text { NMDA receptor antagonists }\end{array}$ & $\begin{array}{l}\text { Antipsychotics targeting } \\
\text { Dopamine and serotonin } \\
\text { receptors }\end{array}$ & Treat the organic problem \\
\hline
\end{tabular}

Table 1: Comparison of conditions with visual perceptual distortions.

prudent use in a short time frame of less than 3 months was found to be effective in this case without resulting in sedative-hypnotic dependence.

It is also relevant to mention that patient's disposition at the time of discharge was a significant factor in the management and treatment of this case. Among other considerations are choice of caregiver and consistent location of residence for patients with CBS. These factors were key to improvement of symptoms documented in this case. As reported above, the exacerbation of visual hallucinations occurred around the period when patient's close relative was unavailable. With this noticeable trend as documented in this case, we thus suggest that symptoms in CBS improve considerably when patients are placed in a structured environment such as an assisted living facility. In this case, the patient was discharged to an assisted living facility where there was 24 hour close monitoring and assistance with activities of daily living.

Treatment of CBS in this patient, including improvement in left eye visual acuity from $20 / 200$ to 20/50 majorly contributed to the resolution of the visual hallucinations. After discharged from the hospital, patient continued to receive brimonidine tartrate $0.2 \%$ and timolol maleate ophthalmic solution $0.5 \%$ per ophthalmologist's recommendations. This supports the fact that the visual changes noted in CBS are the key pathophysiological factor contributing to the syndrome. In CBS there is dissociation of visual perception and sensory input; furthermore, functional magnetic resonance imaging has shown that hallucinations of color, faces, textures, and objects correlate with increased cerebral activity in ventral extra striate visual cortex [20]. A functional MRI was not obtained in this case as it was unavailable at the facility.

Another relevant neuropsychiatric disorder that was considered as a differential diagnosis is Anton Babinski syndrome (ABS), which is characterized by bilateral cortical blindness, anosognosia with visual confabulation. In ABS, radiological imaging shows damage in the occipital lobe as the cause of cortical blindness which differs from CBS as there is no specific brain damage in CBS and insight is preserved [21]. Lhermitte hallucinosis (LH) which is another similar condition to CBS was a differential considered in this case report. Note however that patients with LH usually have vivid visual hallucinations in the absence of a past history of psychosis. The main differentiating points are that LH is directly related to prior history of dementia while CBS is not. Also, in $\mathrm{LH}$, hallucinations are due to ischemic lesions in the cerebral peduncle [22].

While there was CT imaging evidence suggesting bilateral calcification of the basal ganglia, there was no significance in relation to patient's symptoms. Basal ganglia calcifications are often idiopathic in nature and this is known as Fahr's syndrome (FS) [23]. FS usually presents with clumsiness, fatigability, unsteady gait, slow or slurred speech, difficulty swallowing, involuntary movements or muscle cramping [24-26]. Despite the basal ganglia calcification, there were no tremors, involuntary movements or unsteady gait and visual hallucinations resolved without requiring further neurological intervention therefore, there was no direct link with abnormal CT image and patient's presentation.

Lastly, dementia with lewy body can be considered in this case given that the patient has core features including visual spatial orientation impairment and decreased cognitive function however, this is explainable by the presence of visual impairment in terms of post cataract procedure and diminished visual acuity. The absence of core features including the lack of fluctuating cognition, findings of ischemia on CT brain made lewy body dementia less likely [27]. Also, if this case were consistent with lewy body dementia, there would have been some response with Rivastigmine and patient would have exhibited symptoms of Parkinson's when started on dual antipsychotic therapy due to neuroleptic sensitivity in lewy body dementia [27] (Table 1).

\section{Conclusion}

Various neuropsychiatric conditions can produce visual hallucinations but there is particular increase among the geriatric population. Given the complexity of the presentation of such symptoms due to behavioral disturbances including agitation and paranoia among elderly patient, it may be challenging to make an accurate diagnosis without proper history taking and physical examination focused on the visual field examination. We suggested that elderly patients presenting to the ED with visual hallucinations, agitation, paranoia or cognitive decline should receive a visual field testing. Any form of diminished visual field impairment in combination with psychotic-like features among the geriatric patients should prompt physicians to include the differential diagnosis of CBS and other similar disorders as this would help manage the patients with a different approach and more favorable outcome. With the rising population of the geriatric patients in the United States of America, and the co-occurrence of visual field defects including cataracts, we project that there would be an increase in the presentation of similar cases to various emergency departments and psychiatric units. Psychiatrists and all physicians are thus encouraged to 
continue to consider a broad differential diagnoses as listed in this case reports as patients with visual hallucinations do not always respond to antipsychotic agents.

\section{Acknowledgement}

Authors of this manuscript give special gratitude to Dr. Marcos-Gonzalez Sanchez M.D. Ph.D., Director of Clinical and Translational Research at Larkin Community Hospital, for his assistance in the revision of this work.

\section{Ethical Approval}

All procedures and interviews conducted were in accordance with the ethical standards of the responsible committee on human experimentation (institutional and national) and with the Helsinki Declaration of 1975, as revised in 2000.

\section{Limitations}

Electric encephalogram (EEG) was not obtained in this case. Functional MR or MRI were not obtainable in this case.

\section{References}

1. Wan He, Daniel Goodkind, Paul Kowal (2016) U.S. Census Bureau, International Population Reports, P95/16-1, An Aging World: 2015, U.S. Government Publishing Office, Washington, DC.

2. http://www.who.int/mediacentre/factsheets/fs381/en/

3. Harvey P (2002) Common eye diseases of elderly people: Identifying and treating causes of vision loss. Gerontology 49: 1-11.

4. Tune L (2001) Assessing psychiatric illness in geriatric patients. Clin Cornerstone 3: 23-36.

5. Gordon M (2000) Problems of an aging population in an era of technology. J Can Dent Assoc 66: 320-322.

6. Pang $L$ (2016) Hallucinations experienced by visually impaired: Charles Bonnet syndrome 93: 1466-1478.

7. Teeple RC, Caplan JP, Stern TA (2009) Visual hallucinations: Differentia diagnosis and treatment. Prim Care Companion J Clin Psychiatry 11: 26-32.

8. Mocellin R, Walterfang M, Velakoulis D (2006) Neuropsychiatry of complex visual hallucinations. Australian \& New Zealand Journal of Psychiatry 40: 742-751.

9. Jackson ML, Ferencz J (2009) Charles Bonnet syndrome: Visual loss and hallucinations. CMAJ 181: 165-176.

10. Quillen DA (1999) Common causes of vision loss in elderly patients. Am Fam Physician 60: 99-108.

11. https://www.nei.nih.gov/eyedata/cataract

\section{Lindstrom $R$ (2015) Thoughts on cataract surgery.}

13. Tan CSH, Lim VSY, Ho DYM, Yeo E, Ng BY, et al. (2004) Charles Bonnet syndrome in Asian patients in a tertiary ophthalmic centre. $\mathrm{Br} \mathrm{J}$ Ophthalmo 88: $1325-1329$.

14. Merabet LB, Maguire D, Warde A, Alterescu K, Stickgold R, et al. (2004) Visual hallucinations during prolonged blindfolding in sighted subjects. J Neuroophthalmol 24: 109-113.

15. Teunisse RJ, Zitman FG, Cruysberg JR, Hoefnagels WHL (1996) Visual hallucinations in psychologically normal people: Charles Bonnet syndrome. Lancet 347: 794-797.

16. Jan T, Del Castillo J (2012) Visual hallucinations: Charles bonnet syndrome. West J Emerg Med 13: 544-547.

17. Seeman $P$ (2010) Dopamine $D 2$ receptors as treatment targets in schizophrenia. Clin Schizophr Relat Psychoses 4: 56-73.

18. Galimberti D, Scarpini E (2016) Old and new acetylcholinesterase inhibitors for Alzheimer's disease. Expert Opin Investig Drugs 25: 1181-1187.

19. Annicchiarico R, Federici A, Pettenati C (2007) Rivastigmine in Alzheimer's disease: Cognitive function and quality of life. Ther Clin Risk Manag 3: 11131123.

20. Ffytche DH, Howard RJ, Brammer MJ, David A, Woodruff P, et al. (1998) The anatomy of conscious vision: an fMRI study of visual hallucinations. Nat Neurosci 1: 738-742.

21. Chen JJ, Chang HF, Hsu YC, Chen DL (2015) Anton-Babinski syndrome in an old patient: A case report and literature review. Psychogeriatrics 15: 58-61.

22. Talih FR (2013) A probable case of peduncular hallucinosis secondary to a cerebral peduncular lesion successfully treated with an atypical antipsychotic. Innov Clin Neurosci 10: 28-31.

23. Saleem S, Aslam HM, Anwar M, Anwar S, Salee M, et al. (2013) Fahr's syndrome: Literature review of current evidence. Orphanet Journal of Rare Diseases 8: 156.

24. Lehman AF, Lieberman JA, Dixon LB, McGlashan TH, Miller AL (2004) Practice guideline for the treatment of patients with schizophrenia. Am J Psychiatry 161: 1-56.

25. American Psychiatric Association (2007) Practice guideline for the treatment of patients with Alzheimer's disease and other dementias. Am J Psychiatry 164 $5-56$.

26. Menon GJ, Rahman I, Menon SJ, Dutton GN (2005) Complex visua hallucinations in the visually impaired. Arch Ophthalmol 123: 349-355.

27. Gomperts SN (2016) Lewy body dementias: dementia with lewy bodies and parkinson disease dementia. Continuum : Lifelong Learning in Neurology 22 435-463. 